\title{
La convection mixte dans les métaux en fusion
}

\section{Mixed convection in molten metals}

\author{
Jacques $\mathbf{H u e t z}$ \\ Directeur du Groupe de Recherches Thermiques du C.N.R.S. \\ associé à l'École Centrale des Arts et Manufactures \\ Professeur à l'École Centrale des Arts et Manufactures \\ Châtenay-Malabry
}

La convection mixte fait intervenir, comme on le sait, les échanges thermiques dûs à la convection naturelle et ceux qui résultent de la convection forcée. L'importance relative de ces deux processus d'échange se détermine par la valeur du nombre de Froude, quotient du nombre de Reynolds et de la racine carrée du nombre de Grashoff.

Avant d'examiner plus en détail les particularités thermiques des métaux en fusion ou liquides que nous désignerons désormais par $M F$, délimitons ceux des secteurs de l'activité industrielle qui sont concernés par l'écoulement des métaux liquides ; les transferts thermiques auxquels sont soumis ces derniers peuvent être de deux types : - l'anisothermie est créée par les obligations de l'élaboration d'un produit métallique - les $M F$ sont utilisés comme fluides caloporteurs. Précisons davantage en parlant de la sidérurgie et de l'énergétique.

- La sidérurgie où l'élaboration ou l'affinement est le but final impose des niveaux thermiques où le métal est liquide.

- L'énergétique où les $M F$ présentent dans des cas précis des avantages décisifs sur les autres fluides caloporteurs. Néanmoins, des sujétions d'emploi d'ordre extrathermique font limiter leur usage aux cas où ils sont irremplaçables, cas qui peuvent, peut-être d'une manière assez conventionnelle, se répartir sur trois domaines :

- les réacteurs nucléaires à neutrons rapides;

- les applications spatiales où toute machine thermique embarquée ne dispose plus comme source froide que d'un "radiateur" puisque le condenseur habituel manquerait du fluide convecteur pour condenser puisqu'il n'y a plus rien pour convecter. La conception d'un condenseur viable pour cette application doit utiliser le rayonnement des parois vers l'atmosphère et, en conséquence, élever le niveau thermique pour conserver des surfaces radiatives raisonnables. Admettre la température de la source froide vers $800^{\circ} \mathrm{C}$ limite singulièrement la nature du fluide de travail de la machine thermique ;

- enfin, les chaudières solaires à haute température et haute concentration n'ont pas encore trouvé leur voie définitive : celle où un capteur à métal liquide constituerait le choix principal constitue une des possibilités.

Néanmoins, sur le plan technologique, les problèmes thermiques et sidérurgiques sont très différents puisqu'on subit la nature du métal que l'on traite, tandis que l'on peut choisir le caloporteur parmi les métaux à priori les plus adaptés. Mais les distinctions ne s'arrêtent pas là et il peut être utile de rappeler à ce point de vue l'essentiel.

Comme tout fluide, le métal liquide a un contenant et c'est plutôt le couple contenu-contenant qu'il faut envisager.

A ce point de vue, la sidérurgie emploie bien souvent des surfaces libres où des échanges radiatifs se produisent avec les matériaux solides en général très émissifs. Ces surfaces libres peuvent être étendues aux jets de coulée.

Dans un circuit de caloporteur, peu ou pas de surfaces libres générant des transferts radiatifs : quant aux jets, il est préférable de les éviter, la nature des métaux liquides caloporteurs habituels étant peu compatible avec un contact avec l'air ambiant.

Toujours dans l'optique contenant-contenu, une autre différence est évidente et essentielle entre les deux domaines d'application. Dans un cas, le $M F$ se renouvelle constamment. Dans l'autre, contenu et contenant sont liés l'un à l'autre pour une durée voisine de la durée de vie du circuit. Aussi, la charge progressive et lente en produits inévitables et nuisibles croît-elle dans le caloporteur : hydrures, oxydes, etc. Comme par ailleurs les 
différences de température d'un "circuit" sont grandes, des processus insidieux et cumulatifs de dissolution différentielle fonction de la température du lieu, grèvent les circuits de métaux liquides caloporteurs. C'est là un des aspects de la "corrosion" contenant-contenu qui, concernant les $M F$ employés en thermique, a une grande importance.

Ces généralités étant dites, confinons l'exposé à l'emploi des $M F$ comme fluide caloporteur en thermique sans aborder plus avant les processus de corrosion, d'oxydation, de diffusion des gaz sous pression à travers la paroi d'un échangeur eau (ou vapeur) vers le métal liquide, pour ne plus envisager que la mécanique des fluides et le transfert convectif par ce type de caloporteurs. Et d'abord, quels métaux sont-ils des concurrents sérieux vis-à-vis de tous les autres fluides, eau vapeur, fluides organiques ou gaz?

Conventionnellement et de manière quelque peu arbitraire, on appellera métaux liquides ceux dont la température de fusion se place en-dessous de $300^{\circ} \mathrm{C}$ environ : ils sont douze parmi lesquels, en fonction de caractéristiques corrosives ou rédhibitoires, on retiendra cinq : le plomb, le bismuth, le sodium, le potassium, le lithium.

Quelques mésaventures sur le plan de la corrosion du contenant ont pour l'instant éliminé les deux premiers. En fait, ce sont les alcalins qui sont seuls employés malgré leurs défauts dont le principal est l'obligation de les tenir à l'abri rigoureux de toute contami-

\begin{tabular}{|l|c|r|r|r|c|}
\hline Tableau I & \multicolumn{7}{|c|}{ Métaux liquides } \\
\hline & $\theta_{\text {fusion }}$ & $\theta_{\text {ébul. }}$ & $\rho$ & $P_{r}$ & $\rho C_{p} / \rho C_{p \text { eau }}$ \\
\hline$L i$ & 180 & 1317 & 500 & 0,06 & 0,53 \\
\hline$N a$ & 98 & 883 & 850 & 0,005 & 0,27 \\
\hline$K$ & 64 & 760 & 750 & 0,003 & 0,13 \\
\hline$P_{b}$ & 327 & 1730 & 10500 & 0,02 & 0,38 \\
\hline$B i$ & 271 & 1478 & 9500 & 0,013 & 0,33 \\
\hline$H g$ & -38 & 357 & 12800 & 0,01 & 0,42 \\
\hline$N a K$ & 7 & 813 & 820 & 0,01 & \\
\hline \multicolumn{6}{|l|}{ Unités S.l et ${ }^{\circ} \mathrm{C}$} \\
\hline
\end{tabular}

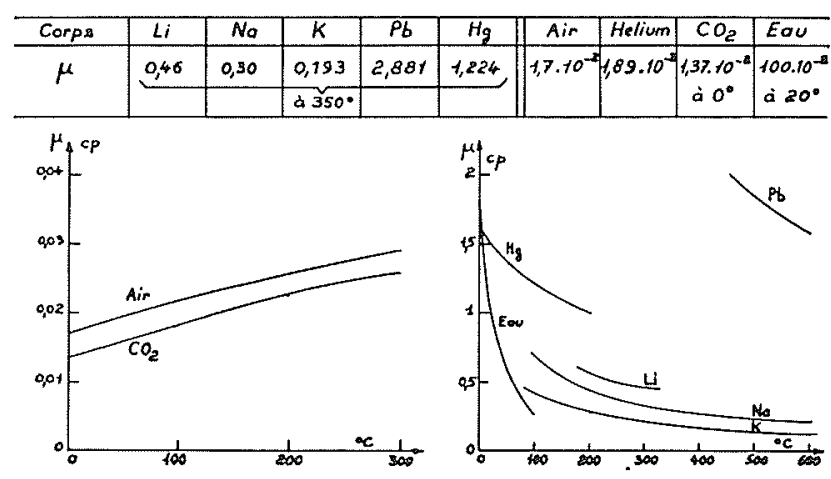

Figure 1 - Viscosité fonction de $T$ en centipoise.

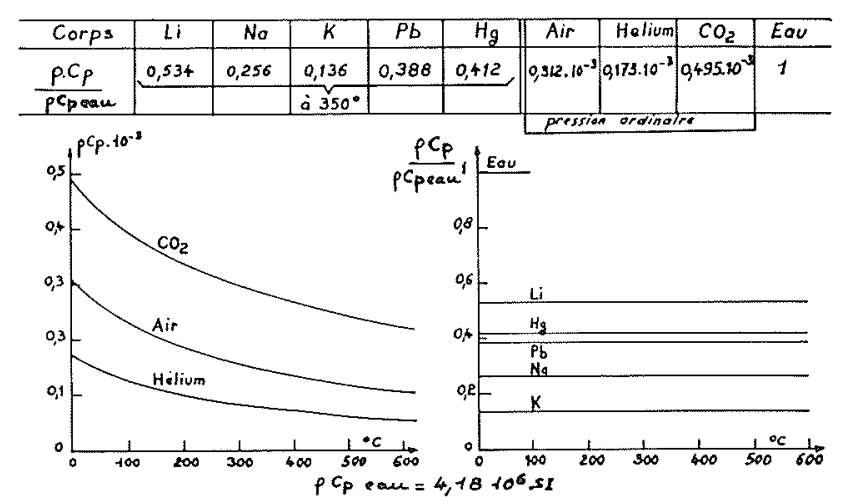

Figure $2-\rho C_{p}$ fonction de $T \mathrm{cal}^{\circ} \mathrm{C}^{-1} \mathrm{~cm}^{-3}$.

\begin{tabular}{|c|c|c|c|c|c|c|c|c|c|}
\hline \multirow{3}{*}{ 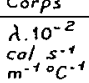 } & $L i$ & $\mathrm{Na}$ & $K$ & $P b$ & $\mathrm{Hg}_{\mathrm{g}}$ & Air & Helium & $\mathrm{CO}_{2}$ & Eou \\
\hline & 0,091 & 0,17 & 0,095 & $0,0.37$ & 0,03 & \multirow{2}{*}{$\begin{array}{l}57.10^{\circ} \\
20^{\circ} \mathrm{C}\end{array}$} & \multirow{2}{*}{$\begin{array}{l}34.10^{-3} \\
\text { i } 0^{\circ}\end{array}$} & \multirow{2}{*}{$\begin{array}{|cc|}55 & 10^{-3} \\
\therefore 1 & 100^{\circ}\end{array}$} & \multirow{2}{*}{$\begin{array}{l}14 a 10^{3} \\
\text { a } 25^{\circ}\end{array}$} \\
\hline & & & $i_{3} 30^{\circ}$ & & & & & & \\
\hline
\end{tabular}

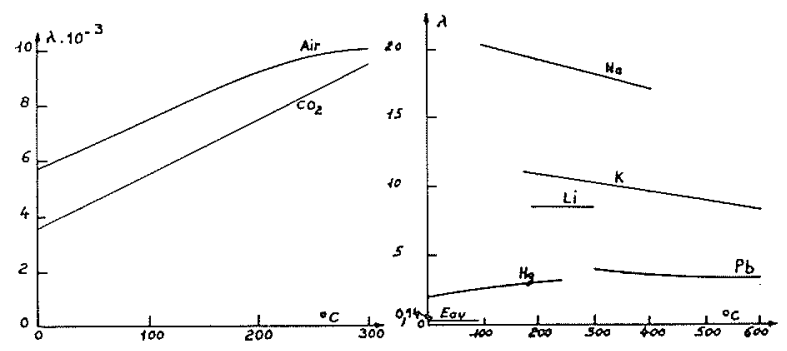

Figure $3-\lambda$ Conductivité fonction de $T$ cal ${ }^{\circ} \mathrm{C}^{-1} m^{-1} s^{-1}$.

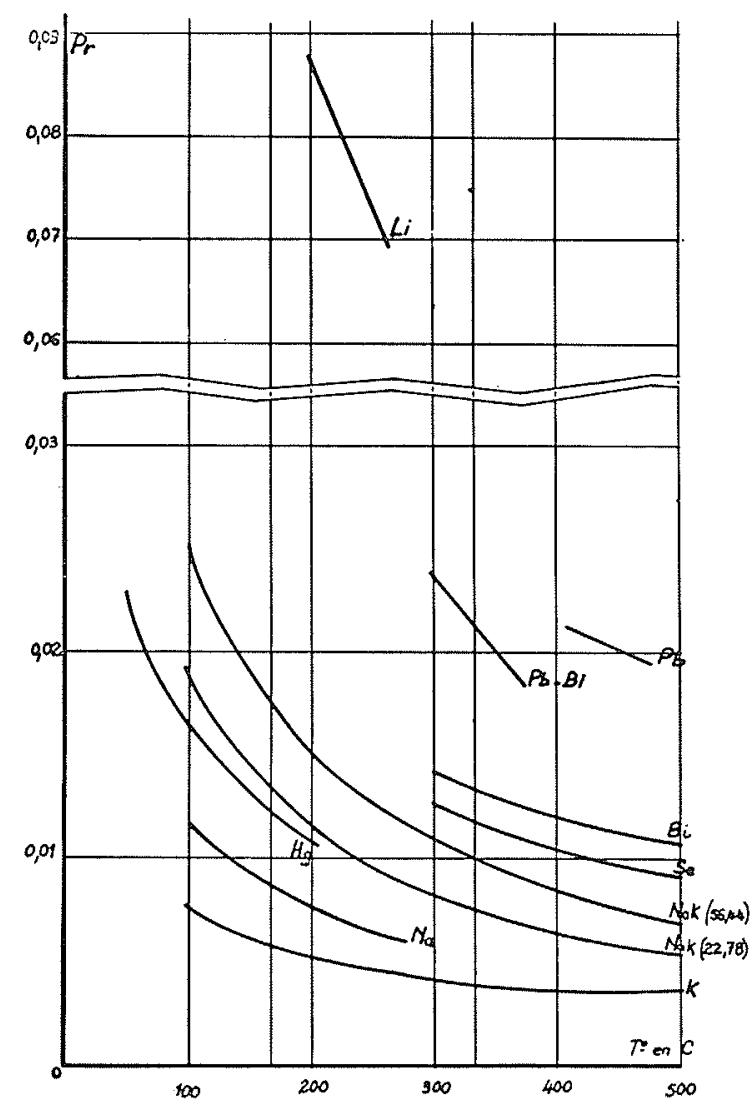

Figure 4 - Nombre de Prandtl. 
nation par l'oxygène atmosphérique ou de la vapeur d'eau. Le tableau 1 donne les principales caractéristiques thermiques des métaux fondus caloporteurs, le $\mathrm{NaK}$ désignant non un eutectique mais un alliage à $56 \%$ de potassium. Mais les ordres de grandeur comparatifs des paramètres thermophysiques ou mécaniques apparaissent sur les figures $1,2,3,4$ où l'on remarquera l'essentiel. Mêmes ordres de grandeur que pour les fluides usuels sauf pour la conductivité $\lambda$ et le nombre de Prandtl $\nu / a=\mu C_{p} / \lambda$. Si l'allure des variations en $\theta$ est commandée par celle de $\mu$ comme pour tous les autres liquides, des valeurs de $\lambda 100$ à 300 fois plus élevées déplacent. le nombre de Prandtl du $M F$ dans le domaine des valeurs inférieures à $10^{-2}$ sauf en ce qui concerne le lithium.

Conséquence immédiate pour l'utilisation des $M F$ :

Si les paramètres qui déterminent l'écoulement isotherme, donc la mécanique des fluides, sont tous du même ordre de grandeur que pour les liquides usuels, rien ne doit distinguer les $M F$ des autres liquides : à ce point de vue, grosso modo, c'est bien ce que l'on constate en effet.

- En convection forcée laminaire, la diffusivité thermique agit proportionnellement à sa valeur sur le coefficient convectif $h$.

- Si on se place dans le cas d'un régime laminaire établi où le Nusselt a une valeur constante 3,66 ou 4,36 pour un tube à température de paroi constante ou à flux surfacique constant respectivement, la conséquence sur $h$ est :

$$
h=\frac{3,66 \lambda}{D_{h}} \text { ou } \frac{4,36 \lambda}{D_{h}}
$$

Les tables de constantes donneront immédiatement:

$$
\frac{h \text { métal liquide }}{h \text { eau }}=\frac{\lambda_{\text {métal }}}{\lambda_{\text {eau }}} \neq 120
$$

Pour la même différence de température, les flux surfaciques sont plus que centuplés.

Autre conséquence : si on compare eau et sodium pour fixer les idées, le gradient axial de la température de mélange soit $\partial \theta_{M} / \partial x$ est donné par :

$$
\Pi R^{2} \rho C_{p} V_{q} \frac{\partial \theta_{M}}{\partial x}=2 \Pi R h\left(\theta_{M}^{-\theta}\right)
$$

soit 250 fois plus élevé pour la même vitesse de circulation (voir le tableau 1 ) et pour des valeurs relatives de $C_{p}$ de l'ordre de 2 ( $C_{p}$ de l'eau ayant la valeur la plus élevée).

La conduction axiale, toujours négligeable pour les fluides habituels, risque pour certaines applications des $M F$ de ne plus l'être. C'est surtout dans le cas de l'écoulement laminaire ou de la convection naturelle que le bien fondé de l'hypothèse classique devra être vérifié.

Si maintenant on examine la convection forcée en régime turbulent, les expressions utilisées par les ingé- nieurs sont comme on le sait de la forme :

$$
\begin{aligned}
& \left|\frac{\tau_{p}}{\rho}\right|=\left(\nu+\epsilon_{m}\right) \frac{\partial u}{\partial y} \\
& \left|\frac{\varphi}{\rho C_{p}}\right|=\left(a+\epsilon_{H}\right) \frac{\partial \theta}{\partial y}
\end{aligned}
$$

Malgré toutes les critiques, fondées sur le plan du principe, qu'il est possible de faire à l'encontre de telles expressions, ce sont bien celles qu'on utilise faute de mieux en définissant des diffusivités turbulentes $\epsilon_{M}$ et $\epsilon_{H}$. On s'y attendait, la croissance de $\epsilon_{M}$ avec le Reynolds et sa variation avec la distance à la paroi sont bien conformes à ce que donnent les théories "classiques". Il n'en n'est pas de même pour $\epsilon_{H}$. L'analogie de Reynolds qui permet d'écrire que, en général $\epsilon_{M}(R e, r)=\epsilon_{H}(R e, p r, r)$ n'est plus valable ici, pour plusieurs raisons dont la principale réside dans le processus physique microscopique de transfert : deux assemblées de particules qui suivent des lois statistiques entièrement différentes, les ions et les électrons, sont responsables du transfert de quantité de mouvement (manifestation de la viscosité) et du transfert d'énergie cinétique décoordonnée (chaleur). En extrapolant une caractéristique valable beaucoup plus pour les métaux purs que pour les alliages, on admettra que les électrons sont responsables du transfert de chaleur, les ions du transfert des quantités de mouvement coordonné. Quoi qu'il en soit des processus physiques, on a en général : $\epsilon_{H} \leqslant \epsilon_{M}$, l'égalité n'étant réalisée que comme limite, quand le nombre de Reynolds croît au-delà des valeurs normalement réalisées dans les écoulements industriels.

Les figures 5 et 6 extraites de Stein [1] illustrent parfaitement ce fait. Si (Fig. 5) une turbulence modérée fait croître $\epsilon_{H}$ à la valeur $\epsilon_{M}$ pour les fluides à nombre de Prandtl voisin de 1 tandis que simultanément $a \ll \epsilon_{H}$ permet de négliger les processus de conduction laminaire devant les transferts turbulents, la diffusivité considérable des $M F$ ne permet plus cette approximation. C'est ce que montre la figure 6 où est portée en ordonnée la valeur de $a /\left(a+\epsilon_{H}\right)$ en fonction de la distance à la paroi. La sous-couche thermique laminaire où $\epsilon_{M}=\epsilon_{H}=0$ et où seule intervient la conduction moléculaire est considérablement plus étendue pour

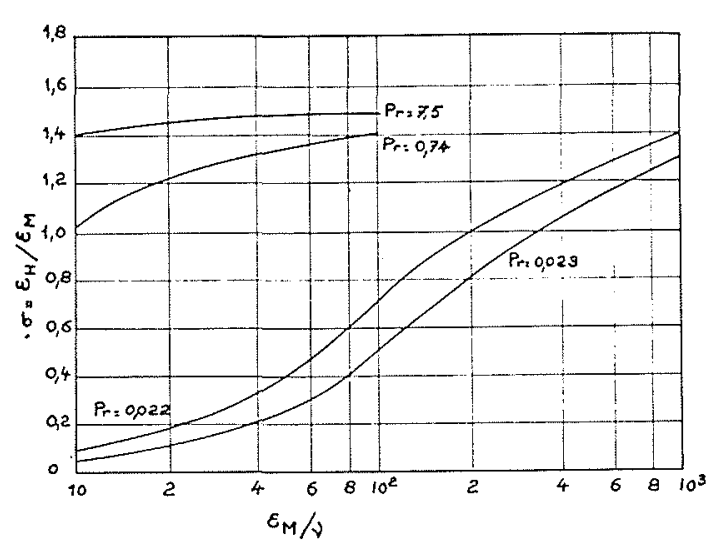

Figure 5 - Rapport des diffusivités turbulentes. 

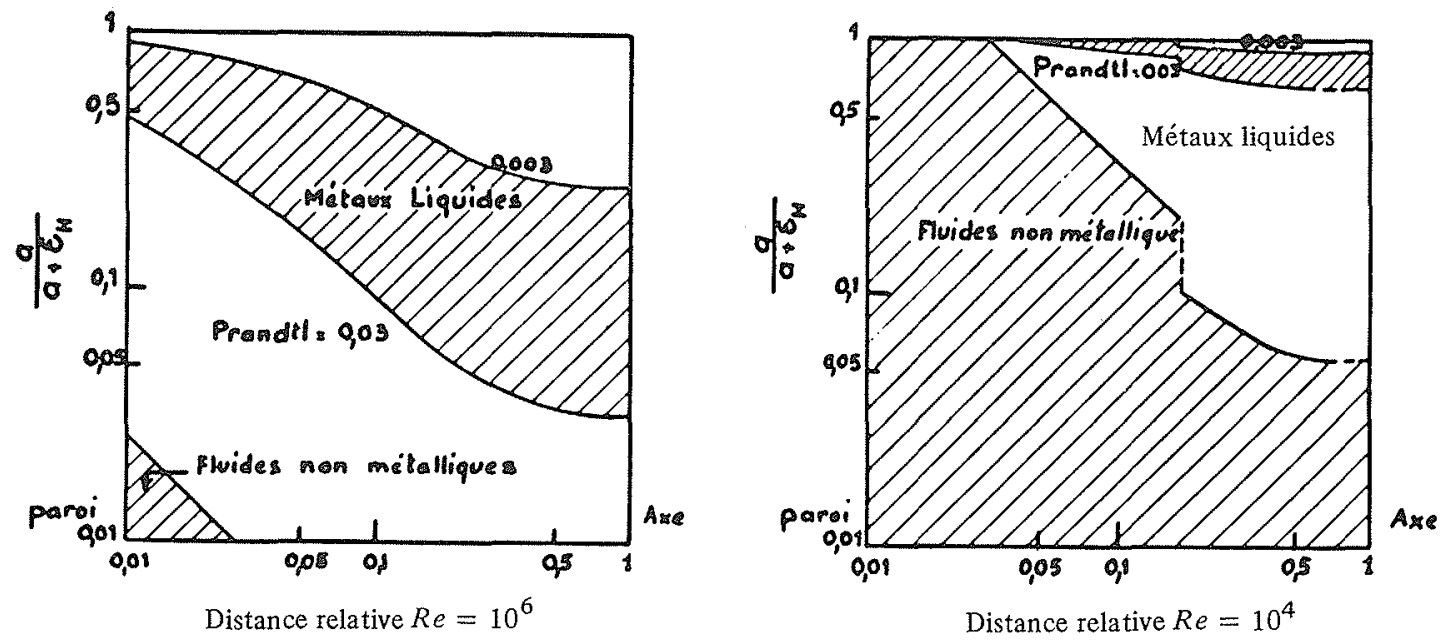

Figure 6 - Influence de la distance à la paroi pour $\epsilon_{H}$.

les $M F$ que pour les autres fluides. La figure comporte deux courbes pour deux valeurs données du nombre de Reynolds.

On peut donc conclure, toujours comparativement aux autres fluides, que si l'écoulement forcé laminaire accroît les transferts dans le rapport des conductivités pour un même champ de température, l'apparition d'une turbulence modérée tend à amoindrir en partie l'avantage considérable que les $M F$ présentent en laminaire par rapport aux fluides usuels. L'augmentation du nombre de Reynolds tend au retour progressif des avantages qui caractérisaient les $M F$ en laminaire sans toutefois parvenir à les récupérer entièrement.

La conséquence est évidente : si le Nusselt qui caractérise le régime laminaire est le même en fluides usuels et pour les $M F$ avec les conséquences sur le coefficient convectif $h$, les lois semi empiriques du régime turbulent (type formule de Colburn) sont faussées par excès et donneront si on les applique, une valeur de Nusselt très nettement surestimée. C'est ce que montre l'évolution du Nusselt en fonction du Péclet pour un tube suivant la formule de Colburn. On 1'a appliquée strictement pour $P r=10^{-1}$ et on obtient des valeurs 50 à 100 fois supérieures à celles de l'expérience ou aux valeurs des expressions adaptées qui sont cependant déjà optimistes quant au transfert (voir Fig. 7).

Pour pallier cette lacune de très nombreuses lois semi-empiriques ont été proposées pour donner $\mathrm{du}$ Nusselt en turbulent pour les $M F$ des valeurs plus réalistes.

Citons par exemple les expressions proposées pour un tube par Skupinsky, Tortel et Vautrey [2] ou par Lyon [3], par Subbotin [4] qui, bien qu'anciennes, donnent des résultats tout aussi corrects que des expressions plus récentes.

$$
\begin{aligned}
& N u=4,82+0,0185 P e^{0,827} \\
& N u=7,0+0,025 P e^{0,8} \\
& N u=5,0+0,025 P e^{0,8}
\end{aligned}
$$

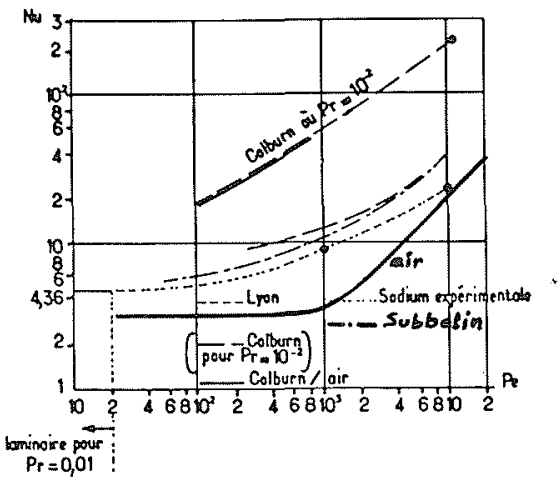

Figure 7 - Formule de Colburn appliquée à l'air et à un métal liquide $: N u=0,023 R e^{0,8} \operatorname{Pr}^{1 / 3}(P e<20)$

Si l'écoulement laminaire ou turbulent forcé des $M F$ se caractérise par des nombres de Nusselt dont on sait calculer la valeur en abandonnant l'analogie de Reynolds, qu'en est-il de la convection naturelle ? En plus des paramètres physiques déjà cités, intervient le coefficient de dilatation cubique $\beta$ dans le nombre de Grashof

$$
G r=\frac{g \beta \delta \theta H^{3}}{\nu^{2}}
$$

A ce point de vue, citons, pour fixer les idées, les ordres de grandeur: (tableau 2).

Le nombre de Grashof à même différence de température est donc du même ordre pour l'eau, pour les métaux liquides et même pour les gaz à haute température si on admet qu'un coefficient 4 est encore dans l'ordre de grandeur. Rappelons que l'on est tout aussi accommodant pour ce qui est des nombres de Prandtl "voisins" de l'unité. En revanche, et toujours à même différence caractéristique, le paramètre attenant à la nature physique du fluide est $\beta / \nu^{2}$ dont les ordres de grandeur figurent aussi dans le tableau 2. 


\begin{tabular}{|l|c|c|c|c|}
\hline \multicolumn{2}{|c|}{$\begin{array}{r}\text { Ordres de grandeur des paramètres physiques } \\
\text { qui interviennent en convection naturelle } \\
\text { pour trois caloparteurs typiques }\end{array}$} \\
\hline & eau à $100^{\circ}$ & \multicolumn{2}{|c|}{$\begin{array}{c}\text { Air à } 700^{\circ} \\
p=20 \mathrm{bar}\end{array}$} & $\begin{array}{c}\boldsymbol{\rho}=1 \mathrm{bar} \\
\mathrm{Na} \\
450^{\circ} \mathrm{C}\end{array}$ \\
\hline$\beta$ & $7,510^{-4}$ & $10^{-3}$ & $10^{-3}$ & $2,410^{-4}$ \\
\hline$\beta / \nu^{2}$ & $1,210^{10}$ & $0,2510^{3}$ & $10^{5}$ & $0,3810^{10}$ \\
\hline$\nu^{2}$ & $6,2510^{-14}$ & $410^{-6}$ & $10^{-8}$ & $6,2510^{-14}$ \\
\hline
\end{tabular}

Là encore ils sont du même ordre pour les liquides, $M F$ compris, mais très différents de ceux des gaz.

Mais si les ordres de grandeur sont les mêmes, c'est aux sources mêmes d'établissement des nombres caractéristiques qu'il faut revenir si on veut là aussi obtenir en fonction du nombre de Grashof des expressions du Nusselt adaptées aux $M F$. Si on conservait ces dernières sans changement, on obtiendrait la même conclusion qu'en convection forcée laminaire : les $h$ convectifs sont, d'un fluide à un autre, pour les mêmes géométrie et différence de température caractéristique, dans le rapport des conductivités. En fait, là aussi, l'augmentation du $h$ convectif quand on passe de l'eau au sodium par exemple est beaucoup plus modérée.

$\mathrm{Si}$ on remonte aux équations adimensionnées grâce auxquelles les résultats d'un cas particulier sont extensibles à de nombreux autres, on s'aperçoit qu'à condition de prendre comme vitesse de référence $V=\sqrt{\operatorname{Hg} \beta \delta \theta}$, le Reynolds et le Péclet, nombres caractéristiques en convection forcée sont remplacés respectivement par $\sqrt{G r}$ et $\sqrt{G r} \operatorname{Pr}$ respectivement, soit deux. nombres caractéristiques. Prendre $G r$ et le produit $\mathrm{Gr} \mathrm{Pr}^{2}$ revient au même. Mais pour un grand nombre de fluides classiques, le nombre de Prandtl est voisin de 1 , en attribuant à l'adjectif "voisin" une marge très étendue. Dans ce cas, la similitude à respecter pour le nombre de Grashof et pour le produit du Grashof par le nombre de Prandtl au carré peut se confondre avec la similitude définie par le seul nombre de Rayleigh produit du nombre de Grashoff et du Prandtl. $\mathrm{Si}$ le nombre de Rayleigh est le même d'un cas à un autre, le Grashof aura à peu près la même valeur et le produit $G r \cdot P r^{2}$ aussi. D'où le succès et le bien fondé des expressions employant le Rayleigh comme nombre caractéristique ; là encore, ces expressions sont inadaptées aux $M F$ et on propose à titre comparatif, pour la plaque plane verticale à titre d'exemple, les formules adaptées en laminaire aux fluides "normaux" et aux $M F$.

$$
\begin{aligned}
& \overline{N u}=0,58(G r \operatorname{Pr})^{0,25}=0,58 R a^{0,25} \quad \operatorname{Pr} \# 1 \\
& \left.\overline{N u}=0,75(G r \cdot \operatorname{Pr})^{2}\right)^{0,25} \quad \operatorname{Pr} \leqslant 0,02
\end{aligned}
$$

soit pour un même Grashof des rapports de Nusselt tels que

$$
\frac{\overline{N u}_{\text {eau }}}{\overline{N u}_{M F}} \# \frac{0,58}{0,75}(100)^{0,25} \# 2,4
$$

$\mathrm{Si}$ on peut détailler et comparer les transferts par convection forcée, qu'elle soit laminaire ou turbulente, et les transferts par convection naturelle, les comparaisons pour la convection mixte deviennent bien évidemment beaucoup plus complexes. Aussi, notre objet n'est-il pas de proposer calculs ou théorie. Mais des expériences paraissent mettre en évidence un écart qualitatif important à la croissance continue du Nusselt qui caractérise le passage du laminaire au turbulent en convection forcée quand, à cette dernière, se mêle la convection naturelle.

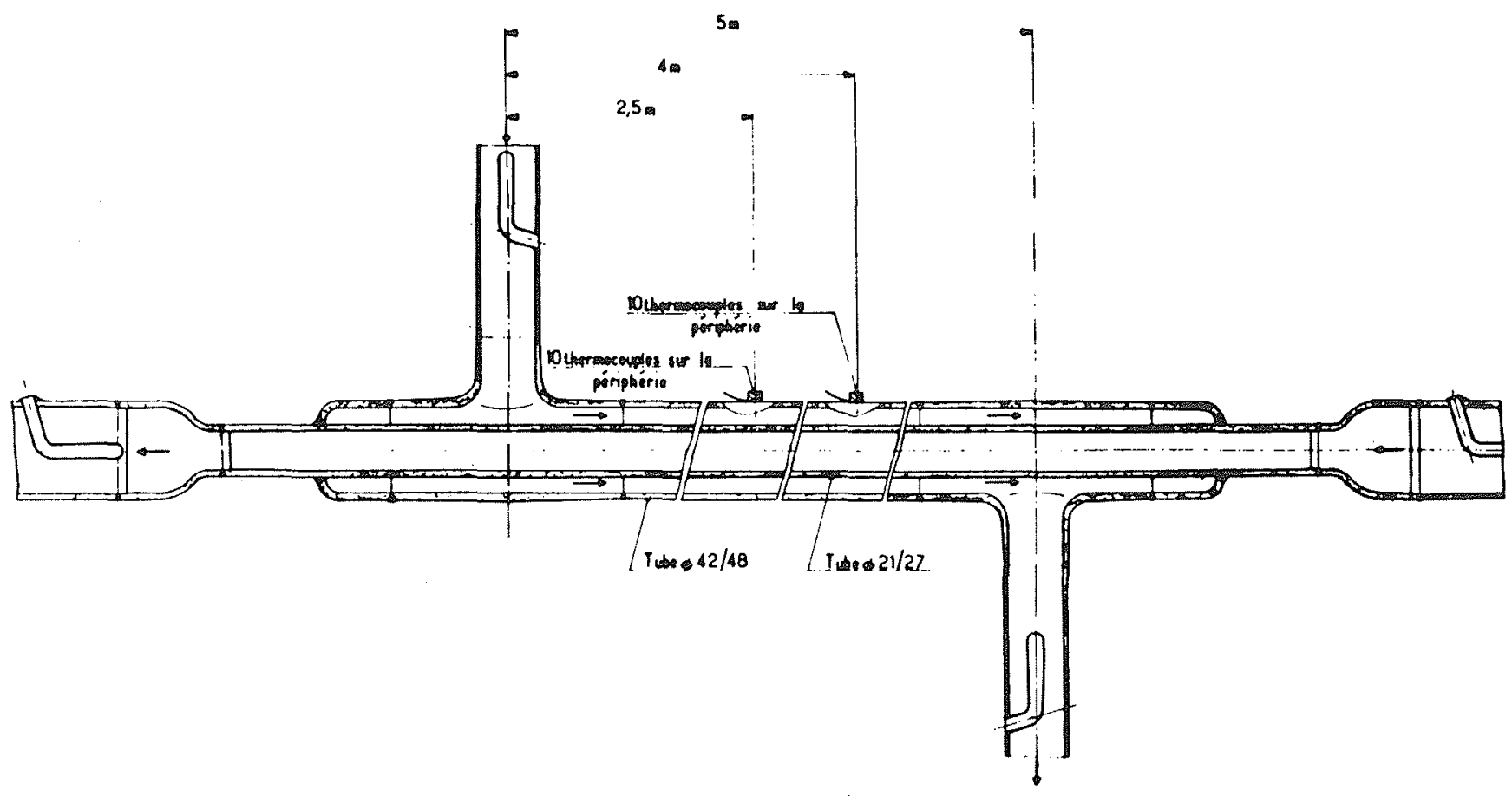

Figure 8- Echangeur expérimental. 


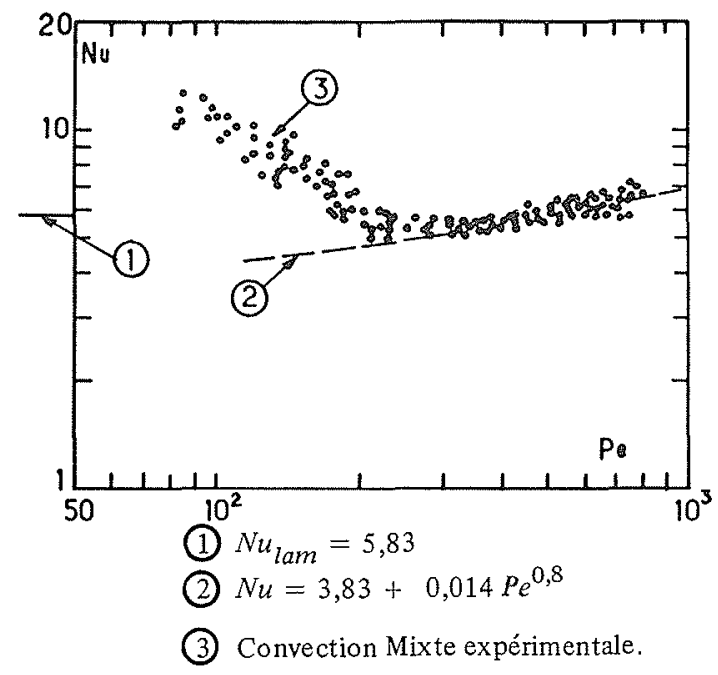

Figure 9 - Aspect du Nusselt en fonction du Péclet pour des turbulences modérées.

Le transfert étudié se situe dans un espace annulaire horizontal dont la figure 8 donne un schéma de principe : l'extérieur est calorifugé ; le transfert avec le fluide du tube intérieur dépend de trois termes : le coefficient convectif du fluide intérieur au tube, la résistance conductive, (ces deux termes sont parfaitement connus), le troisième terme doit être déterminé à partir de mesures expérimentales globales : des couronnes de thermocouples sont disposées dans différentes sections droites dans ce but. Le régime d'écoulement mécanique isotherme s'établit en même temps que commence le contact thermique avec le tube interne.

La détermination expérimentale du coefficient convectif, donc du Nusselt moyen dans l'espace annulaire conduit aux résultats que traduit la figure 9 [6].

En convection forcée fortement turbulente, la valeur du Nusselt concorde bien avec les expressions adaptées aux $M F$ et qui ressortent de formes telles que les formules de Lyon, Subbotin ou autres, adaptées à un espace annulaire.

Mais l'affaiblissement progressif du nombre de Péclet conduit à des résultats bien surprenants. Au lieu de rejoindre progressivement une valeur de 5,6 puisque l'échangeur à contre courant est assez voisin de la condition limite de flux surfacique constant que caractérise cette valeur dans le cas géométrique considéré, on passe par une valeur du Nusselt qui atteint presque le double de la valeur ci-dessus.

Or, c'est là un fait expérimental assez peu connu mais qui a déjá été signalé pour de l'eau [5] avec de mêmes ordres de grandeur pour la croissance du Nusselt. On peut rapprocher cela de tout ce qui a été développé ici pour les liquides sur la convection forcée laminaire des $M F$ et sur les transferts par convection naturelle laminaire ; dans ces deux types d'écoulement, rien ne doit distinguer les ordres de grandeur des nombres de Nusselt caractéristiques et, en conséquence, les transferts sont dans le rapport des conductivités thermiques des différents liquides.

Mais, objectera-t-on, peut-on encore parler de con- vection forcée laminaire pour des nombres de Péclet de l'ordre de 150 , c'est-à-dire des nombres de Reynolds de l'ordre de 7500 où le nombre de Nusselt revient pratiquement aux valeurs qui caractérisent cet écoulement laminaire forcé sans convection naturelle.

Qu'à de telles valeurs du Reynolds la turbulence n'ait pas encore augmenté sensiblement la valeur du nombre de Nusselt de la convection forcée laminaire est absolument conforme à tout ce qui a été montré antérieurement (Fig. 5 par exemple) : une turbulence modérée n'ajoute pas grand chose sur le plan thermique au processus conductif dominant.

Mais comment cette turbulence modérée a-t-elle pu "tuer" une contribution de la convection naturelle très sensible pour des valeurs du Reynolds inférieures? La réponse à cette question n'est qu'une tentative de réponse ou, si l'on veut, une hypothèse.

On remarquera d'abord que, pour un champ thermique donné, la vitesse perpendiculaire à l'axe qui caractérise la convection naturelle est du même ordre pour tous les liquides. Même en laminaire pur, son temps caractéristique reste constant $(t \# H / \sqrt{H g \beta \delta \theta})$ tandis que le temps de parcours d'une longueur finie de tube diminue quand la vitesse, donc le Reynolds, augmente. Au-delà de 2000-2500 pour ce dernier, une turbulence mécanique même faible tend à détruire la stratification en hauteur que la variation en $\theta$ de masse volumique établissait sur la hauteur $H$.

$\mathrm{Ce}$ double mécanisme réduirait donc l'intervention thermique de la convection naturelle avant que la convection forcée turbulente croissante avec le Péclet ne finisse par assurer des transferts thermiques correctement représentés par l'une des trois expressions du Nusselt adaptées aux $M F$ et données ci-dessus.

En conclusion, on peut donc dire qu'une théorie complète de la convection mixte en $M F$ peut reposer sur quelques points fixes auxquels il semble possible de se fier, au moint au titre de première approximation.

- L'écoulement mécanique des $M F$ isothermes ne comporte aucune particularité essentielle par rapport aux fluides caloporteurs habituels.

- Même conclusion pour ce qui concerne la convection forcée laminaire calculable à partir du nombre de Nusselt. Bien entendu, les coefficients convectifs $h$ sont, eux, proportionnels à $\lambda$.

- Même conclusion encore pour la convection naturelle laminaire seule mais en la restreignant pour les comparaisons aux autres liquides.

- Une turbulence croissante en convection forcée augmente les transferts thermiques beaucoup moins vite pour les $M F$ que pour les autres fluides si l'on prend comme référence ce que sont les transferts en convection laminaire dans chaque cas.

- La convection mixte... laisse encore un certain nombre de problèmes à résoudre.

\section{Notations}

$a \quad$ diffusivite thermique $=\lambda / \rho c_{p}$

$C_{p} \quad$ chaleur massique 
$D$

$H$

diamètre hydraulique

relle

$\theta$ ou $T \quad$ températures

$\theta_{p}-\theta_{M}=\delta \theta$ différence de température caractéristique

$R e, P e, P r, G r, N u, R a$, nombres de Reynolds, de Péclet, de Prandtl, de Grashof, de Nusselt, de Rayleigh respectivement

$V \quad$ vitesse ; composantes : $u v w$

$\lambda$ conductivité thermique

$\nu \quad$ viscosité cinématique
$\theta_{M} \quad$ température de mélange

$\theta_{p} \quad$ température de paroi

\section{Bibliographie}

[1] STEIN R. - Dans Advances in Heat Transfer, n 3, 1966.

[2] SKUPINSKI, TORTEL. - Int. J. Heat Mass Transfer, 8, $937,1965$.

[3] LYON R.N. - "Liquid Metals Handbook", NAVEXOS p. 733 (Rev.), 1952.

[4] SUBBOTIN et al. -Atomn. Energ., 13, 380, 1962.

[5]METAIS M.B. - Journées de la Transmission de la Chaleur, I.F.C.E. juin 1961.

[6]PETIT J.P. - Thèse $3^{\mathrm{e}}$ cycle, Paris, 1968.

\title{
Discussion
}

\author{
Président : M. M. BANAL
}

Le Président remercie M. HUETZ et ouvre la discussion.

M. $L E D U C Q$. - Est-ce que les effets électromagnétiques (magnéto-hydrodynamique) sont importants? Il se crée des courants de Foucault qui viennent se superposer aux deux effets de la convection mixte. Quelle est leur importance relative ? Sont-ils toujours négligeables?

M. HUETZ. - En toute rigueur, vous avez parfaitement raison, puisque dans le champ provoqué par les courants qui circulent en général au voisinage des installations industrielles, il y a des effets d'ordre électromagnétique qui se produisent sur le conducteur qu'est le métal lui-même. C'est, du reste, un effet exploité. La mobilité, au sens convection forcée, des métaux liquides peut être obtenue avec des pompes qui créent champ et courant, d'où le déplacement perpendiculaire à l'ensemble cela fonctionne très bien, pour des champs qui sont considérables, vis à vis de ceux que l'on peut accidentellement trouver au voisinage de cuves de métal liquide ou de tubulures. Réciproquement, ce même effet est utilisé en débit-mètre, de manière beaucoup plus fréquente pour provoquer une tension dans un champ magnétique. Il faut peut-être se limiter aux petites installations, et l'on a besoin, pour 2.000 ou 3.000 tonnes de métal liquide, de pompes mécaniques; mais on peut connaître le débit d'une canalisation par le débit-mètre électromagnétique.

Donc, vous avez entièrement raison, ces effets existent qualitativement. Peut-on les préciser quantitativement? Je n'ai pas d'idées précises sur la question. Je pense que, dans le cadre du champ magnétique terrestre, ils doivent être modérés et négligeables.

Le Président. - Les effets électromagnétiques en relation avec les écoulements de métaux liquides sont très intéressants mais ils ne sont pas à notre ordre du jour aujourd'hui. D'ailleurs la Société Hydrotechnique de France a consacré il y a quelques années des séances à leur étude.

M. BONNIN. - Vous nous avez montré le comportement inhabituel, par rapport aux liquides non métalliques, des métaux liquides en convection thermique.

Bien que ce soit hors sujet, pourriez-vous nous éclairer sommairement sur leur comportement spécial en ébullition, compte tenu en particulier de leur chaleur de vaporisation élevée ?

M. HUETZ. - Je ne peux pas répondre à cette question. Mais, jusqu'à présent, les questions de vaporisation du métal liquide ont été envisagées principalement, non sous l'angle du thermicien, non sous l'angle de la chaleur, mais pour des questions de sécurité. La vaporisation des métaux liquides est à peu près normale ; néanmoins, lorsque règne sur eux une pression relativement basse, on constate que les métaux liquides franchissent le point d'ébullition sans qu'il se passe rien, et, brusquement d'une manière un peu instable, il y a un déclenchement de quantité de vapeur assez considérable pour augmenter les pressions dans des zones qu'on n'avait pas prévues et provoquer des incidents.

Dans le cadre du biphasique, des travaux ont été faits sous l'angle de la sécurité ; cela va pratiquement au-delà de $880^{\circ} \mathrm{C}$.

Quand à l'influence de la vaporisation sur le transfert, j'avoue n'avoir pas d'indications à ce sujet.

M. BONNIN. - Dans les travaux que j'ai pu faire, on restait en basse température, donc à basse pression pour explorer le comportement en cavitation. Je visais plutôt le comportement accidentel des réacteurs, mais ce sujet sort visiblement du domaine des travaux que vous avez faits.

M, HUETZ, - Les températures maximales que l'on peut tolérer pour le métal liquide dans des applications nucléaires sont de l'ordre de 550 à $600^{\circ}$. Le sodium est le métal liquide le plus utilisé à l'heure actuelle; or, le sodium à $600^{\circ}$ a une tension de vapeur non négligeable, mais inférieure à la pression atmosphérique ; il n'y a pas de risques.

M. BONNIN. $-600^{\circ}$ est une température encore très faỉble.

Le Président. - Il y a une équipe qui travaille actuellement sur la question du retard à l'ébullition du sodium. Les personnes compétentes, de Grenoble notamment, pourraient apporter quelques informations à ce sujet.

M. $\operatorname{cost} A .-$ Ce phénomène est étudié au C.E.N.G, depuis une dizaine d'années.

Lors des premières expériences, un retard à l'ébullition, "surchauffe", a été mis en évidence. Ce fait était préoccupant pour la sûreté des réacteurs nucléaires utilisant le sodium comme caloporteur car il conduit à une vidange rapide du cœur et par là à un accroissement de la réactivité

Ces premières expériences avaient été faites sur du sodium statique dans un tube chauffé. C'était des expériences fondamentales. Par la suite, des expériences plus "réalistes" ont été faites en convection forcée dans des sections d'essais représentant mieux un assemblage combustible de réacteur. Dans ces conditions, le sodium se met à bouillir sans surchauffe et son comportement en double phase (liquide vapeur) est tout à fait 
semblable à celui de l'eau en ébullition à la pression atmosphérique (les deux fluides ont le même taux d'expansion, c'est-àdire le même rapport entre les densités du liquide et de la vapeur).

M. REMENIERAS. - Au cours des premiers essais de transfert de chaleur dans l'eau effectués au Centre de Chatou (E.D.F.) sur une cellule à deux tubes concentriques analogue à celles décrites par M. HUETZ, on avait tenté d'améliorer le coefficient de transmission en enroulant tout le long du tube central lisse un fil de faible diamètre augmentant sa rugosité (et corrélativement les pertes de charge de l'écoulement). A-t-on repris ce type -d'essai dans le cas des métaux liquides ? Dans l'affirmative peut-on espérer de cet artifice des résultats plus prometteurs que ceux obtenus dans le cas de l'eau?

M. HUETZ. - C'est une expérience qui a été reprise, entre au tres à Cadarache, puisqu'on pouvait ainsi augmenter d'abord la composante de vitesse sur le tube et amorcer des turbulences qui augmentaient le coefficient de transfert.

C'est d'autant plus intéressant comme cas d'étude que le coefficient de transfert convectif croît très lentement quand la turbulence crôtt ; il faut donc des turbulences très actives et virulentes pour augmenter substantiellement le coefficient convectif. Autrement dit, il faut un démarrage.

Et même dans le cas de la conduction pure ou de la convection laminaire, on a un Nusselt constant, quel que soit le liquide. Pour un tube, ce Nusselt serait de l'ordre de 4,36 en convection laminaire, ce qui suppose une géométrie circulaire du tube. C'est vrai pour un métal liquide et aussi pour un gaz. Comme la définition du Nusselt est : $N_{u}=h D / \lambda\left({ }^{*}\right)$ et comme $\lambda$ est 150 fois plus élevé pour un métal liquide que pour de l'eau, h est également 150 fois plus élevé, ceci en régime de convection laminaire.

Malheureusement, quand on prétend l'augmenter par la turbulence, $h$ croît pour les métaux liquides, mais lentement ; il faut, pour obtenir des résultats accentués et observer vraiment un gain thermique qui soit payant, augmenter le Reynolds, donc les pertes de charge, comme pour les autres fluides ayant une viscosité cinématique du même ordre de grandeur, l'eau par exemple, car la viscosité cinématique d'un gaz est bien plus élevé.

Le Président remercie M. HUETz.

* (D. : Dimension géo métrique du tube $: \lambda$ : conductivité) 